\title{
Labyrinthe
}

26 | 2007 (1)

Penser par extraordinaire

\section{L'argument de la filiation, aux fondements des sociétés européennes et méditerranéennes anciennes et actuelles}

Émilie Barraud

\section{(2) OpenEdition Journals}

Édition électronique

URL : http://journals.openedition.org/labyrinthe/1504

DOI : $10.4000 /$ labyrinthe. 1504

ISSN : 1950-6031

Éditeur

Hermann

Édition imprimée

Date de publication : 15 mars 2007

Pagination : 119-124

ISBN : 978-2-9526131-3-2

\section{Référence électronique}

Émilie Barraud, «L'argument de la filiation, aux fondements des sociétés européennes et méditerranéennes anciennes et actuelles », Labyrinthe [En ligne], 26 | 2007 (1), mis en ligne le 01 avril 2009, consulté le 21 avril 2019. URL : http://journals.openedition.org/labyrinthe/1504 ; DOI : 10.4000/ labyrinthe. 1504 


\title{
L'ARGUMENT DE LA FILIATION, AUX FONDEMENTS DES SOCIÉTÉS EUROPÉENNES ET MÉDITERRANÉENNES ANCIENNES ET ACTUELLES
}

\author{
Émilie BARRAUD* \\ emiliebarraud@yahoo.fr
}

Un colloque international et pluridisciplinaire a rassemblé les 4,5 et 6 octobre 2006 au Collège de France des anthropologues, juristes, historiens et philosophes spécialistes des questions de parenté. La rencontre, inscrite dans la continuité d'un précédent colloque consacré à l'alliance ${ }^{1}$, portait cette fois sur «L'argument de la filiation, aux fondements des sociétés européennes et méditerranéennes anciennes et actuelles».

L'objectif premier des organisateurs était de construire un objet commun par l'examen des différents usages de la notion de filiation - ce à quoi renvoie, dans l'intitulé du colloque, le terme «argument». Par ailleurs et au-delà de la question des usages savants, l'enjeu du débat visait les représentations et les modes de construction indigènes de la filiation, lesquels ont notamment conduit à la question (aux limites toujours mouvantes) de l'articulation du biologique et du juridico-social.

Pour traiter de l'argument de la filiation, les organisateurs ont structuré l'exercice en cinq séances de quatre interventions. Les trois séances initiales ont porté sur des textes fondateurs. La première proposait un retour aux origines par l'examen des récits de fondation, partant de l'étude

\footnotetext{
*Étudiante en anthropologie à l'université Aix-Marseille 1 (université de Provence), Émilie Barraud est l'auteur d'un diplôme d'études approfondies intitulé Approche anthropologique du passage à la maternité adoptive, dans le contexte de l'adoption internationale (2004); elle étudie actuellement, dans le cadre de ses recherches de doctorat, le parcours de certains Français (couples mixtes ou binationaux algériens et marocains) qui recueillent un enfant au Maroc et en Algérie par voie de tutelle légale (kafala).

1. Et qui a fait l'objet d'une publication : Pierre Bonte (dir.), Épouser au plus proche, inceste, prohibitions et stratégies matrimoniales autour de la Méditerranée, éd. de l'EHESS, 1994, 488 p.
} 
des processus de nomination et de génération des divinités grecques et romaines (à l'appui d'une lecture des théories de Hermann Usener dans les Götternamen, 1896). Cette approche linguistique fut le moyen de préciser le moment dans l'histoire où les noms ont pris une valeur patronymique, et où il fut nécessaire d'attribuer un père à Zeus. La séance permit aussi de mieux comprendre, à partir d'une chronique royale mésopotamienne (2200 avant notre ère) et d'un récit légendaire sur le premier roi supposé de l'humanité, le schéma héréditaire qui gouvernait les régimes de succession dans les monarchies mésopotamiennes. D'autres contributions ont exploré, à l'aune des récits adamiques, la genèse de l'être humain dans les traditions musulmanes (processus de sexuation du premier homme, passage de l'androgynie à la filiation), ainsi que la création des hommes et de leurs apparentements dans le système touarègue. Chaque système ainsi étudié affirme avec force le caractère construit de la filiation. À l'issue de cette séance, le principe de la filiation s'entend incontestablement comme une construction artificielle utile, voire nécessaire, pour penser le monde et l'ordonner.

La deuxième séance proposait des relectures de systèmes théologiques, montrant l'importance du lien entre la notion de filiation et le champ du sacré. Elle s'ouvrit sur une étude des formes de la parenté homérique, à partir des généalogies que l'Iliade représente brandies avec fierté par les guerriers avant chaque combat. Une deuxième communication, par l'exploration de certains cas d'établissement de filiations au vu du corpus normatif du judaïsme rabbinique, permit de souligner l'importance qu'accordent les traités talmudiques à la paternité naturelle, donc au critère biologique, dans l'établissement de la filiation. À la lumière de la littérature juridique musulmane du $\mathrm{x}^{\mathrm{e}}$ siècle, la réflexion s'est prolongée, questionnant les causalités enchevêtrées (d'ordre biologique et juridique) des empêchements matrimoniaux, et révélant alors une double conception de l'interdit de l'inceste, reposant pour l'une sur l'acte sexuel et pour l'autre sur le lien matrimonial. Enfin, la séance s'est achevée par l'étude des processus de construction de parentèles ${ }^{1}$ dans les traditions chrétiennes, à mi-chemin de représentations relativement contradictoires. En effet le sang, élément structurant de la parentèle et puissant marqueur généalogique, déterminait l'identité filiative de la personne avant d'être supplanté par l'évènement matrimonial, ce qui aboutit à une redéfinition

1. Champ des alliances légitimes au sein des populations chrétiennes. 


\section{L'argument de la filiation...}

du groupe d'appartenance. Au total, ces études de textes religieux ont mis en évidence une sorte de tension entre filiation et alliance (sur laquelle se fondent les configurations étudiées), ainsi qu'un tiraillement entre le biologique et le juridico-social dans l'établissement et la définition de la filiation.

Prenant également appui sur les textes fondateurs, la troisième séance entendait revenir sur les langages de la parenté et la dynamique des groupes. Pour comprendre comment était assurée la transmission générationnelle, la première intervention a porté, à la lumière des religions monothéistes, sur le genre de Dieu, sur la notion de succession positionnelle (lorsqu'un personnage, humain ou divin, occupe simultanément toutes les positions de la parenté) et sur la prédestination sacrificielle du fils aîné dans la plupart des textes. D'autres, en s'appuyant sur les textes de l'Antiquité classique, ont mis en évidence le fonctionnement des groupes de filiation athéniens où la polis est mère commune, où l'acte cultuel s'inscrit dans l'hérédité et où les modes d'affiliation se recoupent tant ils sont multiples; l'étroite association du politique, du religieux et du parental questionne le rôle des groupes de filiation dans une société qui accorde une place importante à la parenté naturelle. Enfin, une analyse du personnage mythique de Romulus a permis d'interroger l'adoption romaine, ou adrogation, à partir d'un cas-limite, à l'exact opposé de la pratique romaine courante (Romulus se donne en adoption à une femme et s'attribue onze frères adoptifs). Au terme de cette troisième séance et au regard des précédentes, il apparaît clair que dans la plupart des contextes étudiés, les représentations locales donnent une définition essentialiste de la filiation (identité transmise en ligne masculine par le sang des générations précédentes) bien que des logiques cognatiques (logiques de transmission indifférenciée par les hommes et les femmes) soient en œuvre dans la constitution des groupes. Les unités parentales présentent un caractère instable puisque, de fait, elles se constituent aussi par l'alliance et sont donc assujetties à des variations continuelles induites par les pratiques matrimoniales.

Alors que la quatrième séance privilégiait l'étude du «fétichisme de la parenté ${ }^{»}$, c'est-à-dire des processus d'attribution de sens permettant

1. Pour de prosaïques raisons de place, nous ne donnons ici qu'un bref aperçu des principales thématiques abordées : stoïcisme et engendrement, théologie médiévale et filiation, filiation légitime dans l'Italie moderne, politique et filiation dans les démocraties actuelles (analyse du rôle des rapports de filiation dans l'élaboration d'une légitimité). 
de légitimer l'ordre de la parenté, voire l'ordre social, l'ultime séance visait plus spécifiquement à appréhender certaines évolutions contemporaines. À partir d'observations ethnographiques, à propos notamment des procréations médicalement assistées en Égypte ou des dons d'ovules en Espagne, de la prohibition de l'adoption en Islam, de la notion d'illégitimité en Norvège, de l'accouchement anonyme ou de l'adoption plénière dans le droit français actuel. Ont alors été débattues les nouvelles problématiques engendrées par les progrès de la médecine occidentale, les rapports étroits et bien souvent contradictoires entre le biologique et le social, et les remises en question du droit dans la théorisation de la filiation. Les pratiques contemporaines de banque de lait, de don de sperme et de sang, et d'insémination en terre d'Islam sunnite interrogent des logiques où les substances (sang, sperme, lait) sont conçues comme support de liens de parenté mais où, paradoxalement, la filiation est avant tout sociale (puisque la détermination juridique de la paternité se fonde sur le lien matrimonial). L'analyse des procréations médicalement assistées en Espagne révèle le paradoxe d'une reproduction qui se biologise alors même que la filiation repose davantage sur le critère de volonté. La pratique adoptive, notamment l'adoption plénière, où le lien de filiation est exclusif, mène à un constat similaire: si l'on observe dans nos sociétés contemporaines une pluralité de configurations de parenté, l'émergence d'un modèle électif fondé sur le choix, et une certaine déconstruction du biologique comme critère de définition du lien filial, en revanche, la construction sociale de la parenté se maintient comme une métaphore contrainte par les données biologiques. Car l'adoption plénière reflète notre modèle culturel de reproduction, «le modèle généalogique», lui-même calqué sur le phénomène biologique de la reproduction sexuée. Le paradoxe se résume ainsi : l'adoption plénière est l'exemple d'un désinvestissement du biologique au profit du critère de volonté, bien qu'elle porte en triomphe la norme de l'exclusivité en se calquant sur le modèle généalogique. Et de telles transformations impriment une complexité croissante au droit de la filiation: l'anonymat des dons et de l'accouchement, les nouvelles formes d'adoption, les revendications des couples homosexuels et des personnes célibataires, les procréations médicalement assistées et la gestation pour autrui mettent sérieusement à l'épreuve le droit français, contraint à la fragmentation.

Ainsi, le colloque a été l'occasion d'une réflexion sur les façons plurielles et culturellement déterminées dont est créée, construite et 


\section{L'argument de la filiation...}

légitimée la filiation. La question des usages de la filiation, usages métaphoriques et usages performatifs, fut privilégiée aux dépens d'autres enjeux traités sans plus d'insistance, tels que l'ordre du masculin et du féminin, et la fonction de la filiation dans la construction du lien social. En revanche, nous avons pu saisir à plusieurs reprises mais sans pour autant qu'il en résulte un schéma unique, une même tension qui procède de l'articulation entre filiation et alliance dans des contextes très différents. Par ailleurs, le rapport biologique/social fut largement débattu. De nombreuses communications ont démontré la nécessaire coïncidence entre le critère social et le critère biologique dans l'établissement et la légitimation de la filiation.

Un constat s'impose clairement à l'issue des débats et rend compte d'une mutation dans la façon de penser la filiation. Peu d'intervenants ont eu l'usage de supports techniques (tels que les schémas de parenté) autrefois récurrents dans l'exposé des communications sur la parenté. Cette observation rend manifeste le changement opéré dans l'appréhension de l'objet: les intervenants ne prétendaient pas amorcer la réflexion à partir du concept de filiation tel qu'il a été construit par les anthropologues entre les années 1920 et 1960 - concept fondé sur le postulat de l'universalité de la méthode généalogique, et sur l'idée que seule la filiation unilinéaire est susceptible de constituer des groupes discrets ${ }^{1}$. Eu égard à cette théorie consacrée de la filiation, le présent colloque traduit un net glissement dans la manière d'aborder la question: la primauté a été donnée ici aux questions des origines de la filiation, de ses fondements épistémologiques et de la manière dont elle combine le biologique et le sacré; la question autrefois incontournable du groupe s'est peu posée.

Ce changement observé dans l'appréhension de l'objet «filiation» n'est pas étranger au flou conceptuel qui, au cours du colloque, a été maintenu autour de cette notion - flou qu'il semble convenu d'accepter sans le juger regrettable, dans la mesure où il maintient une valeur heuris-

\footnotetext{
1. Le concept figure à l'état naissant dans l'ouvrage de Henry Sumner Maine, Ancient Law (1861). Il a ensuite été affiné par les premiers théoriciens de la parenté: John Ferguson McLennan, Johann Jakob Bachofen, et surtout Lewis Morgan (Systems of Consanguinity and Affinity of the Human Family, 1871). Par la suite, nombreux sont ceux dont les études sur la parenté ont contribué à définir une anthropologique de la filiation: citons Julian Pitt-Rivers, Edmund Leach, Alfred L. Kroeber, Alfred Radcliffe-Brown, E.E. Evans-Pritchard, Meyer Fortes ou encore George P. Murdock, Claude Lévi-Strauss et Rodney Needham.
} 
tique. Placés en situation de recomposition conceptuelle, les participants au colloque ont notamment reconnu lors du débat final l'importance du critère démographique propre à la sociologie, de cette relation au calcul dont l'anthropologie s'est fréquemment affranchie: un langage des nombres qu'il apparaît désormais indispensable d'introduire au sein de la réflexion. Par ailleurs, la discussion a montré que l'objet «filiation» avait été dans la plupart des communications construit par ses limites: ce sont en effet principalement des situations marginales qui furent examinées. Le point de départ n'était donc pas un concept établi, mais bien une notion sociologique envisagée par ses marges et ses divers usages.

C'est ainsi qu'au croisement de méthodes réflexives multiformes autour de la filiation, anthropologues, historiens, philosophes et juristes, dans la construction et la définition par ses frontières d'un objet commun, ont su étendre le champ d'analyse tout en le renouvelant. 